
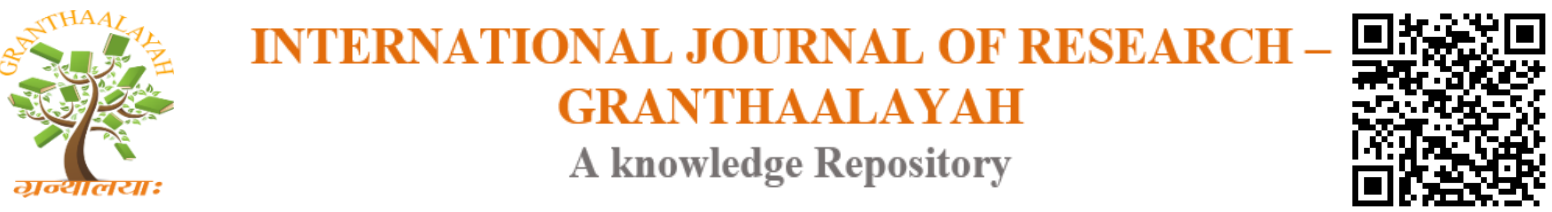

Science

\title{
PERSONAL FACTORS AND THEIR PERSPECTIVE IN SOCIAL MEDICINE AND REHABILITATION
}

\author{
Wolfgang Seger ${ }^{1,2,3}$, Sabine Grotkamp ${ }^{1,2,3}$, Wolfgang Cibis ${ }^{3,4}$ \\ ${ }^{1}$ Health Advisory Board of Social Health and Long-Term-Care Insurances in Lower Saxony \\ (MDKN), Hannover, Germany \\ ${ }^{2}$ Medical Expert Advisory Board of the Federal Rehabilitation Council, \\ Frankfurt, Germany \\ ${ }^{3}$ German Society for Social Medicine and Prevention (DGSMP), \\ Hannover, Germany \\ ${ }^{4}$ Federal Rehabilitation Council, Frankfurt, Germany
}

\begin{abstract}
Motivation / Background: A broad and common understanding of the nature of Personal Factors is deemed to be necessary to gain and assess a comprehensive perspective regarding an individual's health condition and accurately allocate social and medical benefits and interventions. Personal Factors have an impact on the functioning of individuals as facilitators or barriers. They play an essential role in the International Classification of Functioning, Disability and Health (ICF). To date, the World Health Organisation has not classified Personal Factors for global use.
\end{abstract}

Method: A consensus group representing a broad spectrum of medical and social security professionals, research societies together with representatives of self-help organisations developed and published a proposal to classify relevant Personal Factors.

Results: To face ongoing discussions misunderstandings are addressed, critics and suggestions balanced and positions created with pointed explanations and supplemented literature. Core messages summarise each position in a condensed form.

Conclusions: Personal Factors may exert a powerful influence on the goal definition, goal achievement and type, complexity and length of necessary medical, social or rehabilitation benefits. Transparency, explicitness, fairness (standardisation) and the possibility for wellfounded claims comprise additional arguments for reporting Personal Factors. They may be crucial to reach the goal of the highest degree of participation considering individual resources.

Keywords: Functioning; ICF; Personal Factors; Insurance Medicine; Social Medicine; Social Benefits; Participation.

Cite This Article: Wolfgang Seger, Sabine Grotkamp, and Wolfgang Cibis. (2017). "PERSONAL FACTORS AND THEIR PERSPECTIVE IN SOCIAL MEDICINE AND REHABILITATION." International Journal of Research - Granthaalayah, 5(7), 374-389. https://doi.org/10.29121/granthaalayah.v5.i7.2017.2144. 


\section{Introduction}

The International Classification of Functioning, Disability and Health (ICF) of the World Health Organisation (WHO) aims to establish a common language for describing health and health related conditions to improve communication between different users, such as health care workers and individuals with disabilities [1]. The domains contained in the ICF are described from the perspective of the body, individual and society in two components: 1 body functions and structures, which describe the actual anatomy and physiology/psychology of the human body, and 2 activities and participation, which describe an individual's functional status, including communication, mobility, interpersonal interactions, self-care, learning, and application of knowledge, with interactions between the components and functioning and disability represented as umbrella terms (Fig. 1).

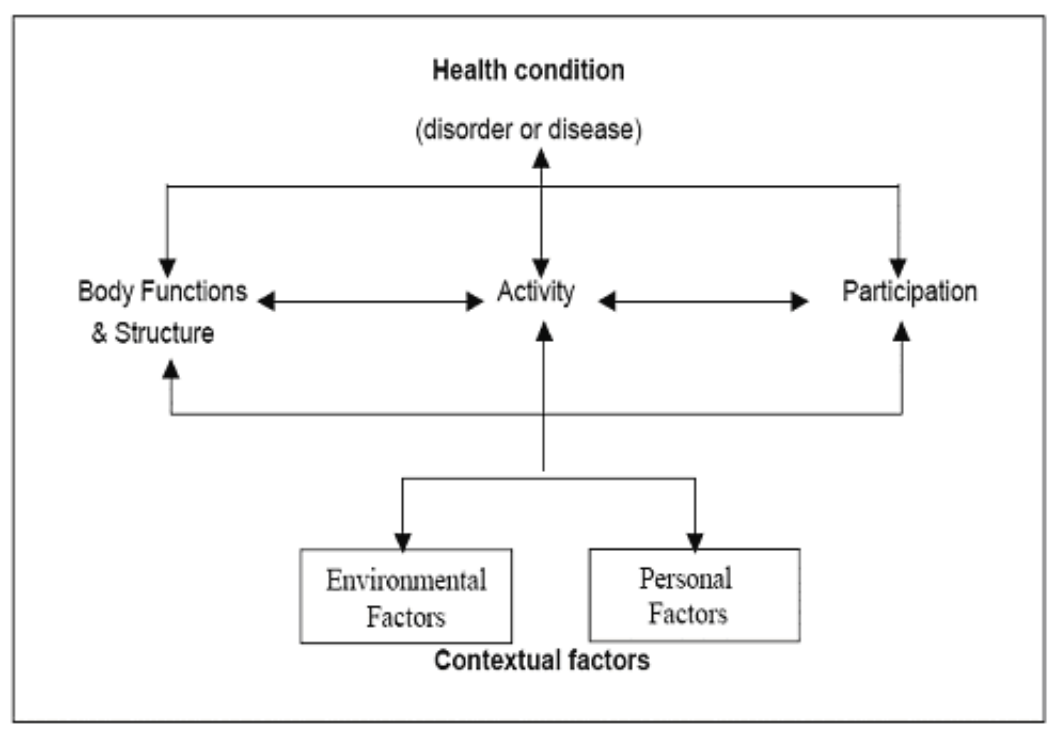

Figure 1: Interactions between the Components of the ICF (WHO 2001)

Environmental Factors and Personal Factors may have an impact on all constructs and may therefore impact an individual's health and health-related states, which are negatively referred to as "barriers" or positively referred to as "facilitators". Personal Factors may include "gender, race, age, other health conditions, fitness, lifestyle, habits, upbringing, coping styles, social background, education, profession, past and current experience (past life events and concurrent events), overall behaviour pattern and character style, individual psychological assets and other characteristics". Personal Factors are attributes of an individual; they are not components of health conditions or health states.

Physicians who practice Social Medicine in health insurances, pension funds or employment agencies have the task of assessing the nature and extent of disturbances in health, their impact on bodily functions and structures and the individual impairment or resource activating effects on functioning, activities and participation in all life situations. This information may result in recommendations regarding sociomedical benefits tailored to suit personal needs, which simultaneously consider the determining framework of the respective insurance or social security systems. This analysis must respect the individual life situations of the individuals involved [2]. 
It is a challenge not only to independently analyse the disease generated impairments but also identify the personal restrictions or personal resources that may help to strengthen participation. A structured, standardised and transparent list of Personal Factors that comprise all sociomedically relevant factors of potential influence on an individual's functioning may support physicians who practice Social Medicine. This framework would facilitate consideration of the background information regarding an individual's social and medical histories and help identify the potential influences of inhibiting or facilitating Factors. This may have an impact on the selection of appropriate and potentially successful medical interventions and social benefits, a process which may have substantial effects on the aspired outcome level of participation as well as on the estimated costs of probably useful or useless interventions. Moreover, this list would only be useful as background information, not as a manual to follow flavishly from A to Z. The selection of Personal Factors from a list must only be considered as useful in a context with potential influences on a particular sociomedical problem at the particular time.

\section{The functioning of a person is classified, not the person!}

The ICF working group of the of the German Society for Social Medicine and Prevention (DGSMP) has compiled and published a draft for the classification of Personal Factors primarily for the German language area (Germany, Switzerland, Austria) [3,4]. It is the objective of this paper to push, maintain and advance the discussion regarding Personal Factors, in general, in the context of social and insurance medicine.

\section{Methods}

The members of the working group who aimed to define and cluster a set of Personal Factors originated from three countries (Germany, Switzerland, and Austria). They represented a broad spectrum of medical and social security professions who practice in rehabilitation centres, hospitals, social and insurance agencies or different health advisory boards. Included were members of several different research societies (social medicine, physical and rehabilitation medicine, occupational and environmental medicine, and neurotraumatology), as well as specialists in social security law and politics. We appreciate the support of representatives of self-help organisations and diverse patient associations. Most members had been previously engaged in the preceding process of preparing a list of Personal Factors. The research design, participants and setting, data sources and collection, criteria for selection, data credibility and trustworthiness checks, procedure, data analysis, ethical considerations, formal decision making and review process, including cross-validation and discussions regarding unnecessary and missing factors, have been previously described [3].

The consensus process resulted in Personal Factors classified into 72 categories and arranged in six chapters as described in table 1.

Table 1: Synopsis of Chapters that describe Personal Factors [3]

Structure of Chapters for Classification of Personal Factors

1 General Personal Characteristics: Age, Sex, Genetic Factors

2 Factors related to an Individual's Physique 


\begin{tabular}{l}
3 Mental Factors, such as Personality Factors and Cognitive and Mnestic Factors \\
\hline 4 Attitudes, Basic Skills and Behaviour Patterns \\
\hline 5 Immediate Life Situation and Socioeconomic / Sociocultural Factors \\
\hline 6 Other Health Factors \\
\hline
\end{tabular}

Picking up ongoing discussions after publication of the draft of a classification of Personal Factors and correspondent presentations in scientific meetings, the working group decided to address misunderstandings perceived. Critics and suggestions from other experts as well as from literature were balanced and included in discussions during several subsequent meetings. Consensually positions were created with pointed explanations and supplemented literature. Core messages summarise each position in a condensed form.

\section{Personal Factors of the ICF are Important for Many Sociomedical Issues}

Personal Factors may act as barriers and negatively interfere with participation. For example, memory factors are specific mental factors, which involve memory, storing information, processing, recall and retrieval. They facilitate the transfer of a patient to a front service workplace area, whereas the lack of retentivity may impede schooling. Moreover, a high reliability for the intake of remedies may be advantageous for therapeutic results, whereas unreliability may urge consideration of the individual prospect of success for the allocation of the best suited vocational rehabilitation benefits; proaction is a necessary skill to develop the motivation to act, plan actions and proceed expediently. These skills may improve the selfmanagement of patients with Diabetes Mellitus, whereas the lack of these skills may endanger home dialysis.

Personal Factors may have an individual impact on functioning; thus, they must be considered as part of a comprehensive evaluation of an individual's disability of relevance for the particular issue. The adequate consideration of Personal Factors is of importance during the entire rehabilitation process, for example, for the appraisal of the individual's rehabilitation potential, the formulation of accurately fitting rehabilitation goals and the recommendation of adequate rehabilitative procedures. For all individual medical activities and interventions, Personal Factors must be considered to achieve the highest level of success for therapeutic interventions by respecting and individually adjusting the type, length, extent, initiation, performance and end of procedures. Personal Factors are also necessary to evaluate the potentials to prevent the loss of a job or facilitate reintegration into employability related to a health problem. In this case, it is inevitable to appropriately and comprehensively assess an individual's positive or negative work capacity. Personal Factors may be the crucial factors that have an impact on a positive change in the long run by applying to a concrete workplace or the common labour market. Therefore, Personal Factors are also important for Occupational Medicine. 


\section{Attention towards Personal Factors Promotes the Idea of Activating Personal Resources to Overcome Participation Restrictions}

A classification of Personal Factors facilitates the systematic identification and assessment of individual-related influencing factors to initiate necessary, promising and potentially successful procedures and to discuss them in dialogue with the client / patient.

Personal Factors are helpful for two different approaches:

1) To assess the functioning of an individual integrated with its interdependencies (resource oriented and deficit oriented); thus, abstinence from alcohol because of belonging to a group with specific religious convictions may support a healthy way of living and promote the rehabilitation of a patient who suffers from liver disease. In contrast, belonging to a group that denies necessary blood transfusions when removing a liver tumour may be life threatening.

2) To appropriately assess the allocation of medical and / or social interventions (resource oriented and deficit oriented); thus, a positive attitude towards intervention and healthrelated assistance in conjunction with treatment compliance may support or enable surgical procedures, the use of prosthetics, medication, psychotherapy or rehabilitation. In contrast, a definitive negative attitude towards the use of a wheeled walker / rollator may lead to social isolation.

For the accurate identification of promising, fitting, social, medical, rehabilitative and workplace-oriented benefits, procedures or interventions, Personal Factors must be identified by their probable effects, i.e., whether they are facilitating or inhibiting. The realisation of diagnosis-, intervention- or ICF-based clinical and social pathways is considerably promoted if participation goals are delineated by considering all ICF-components, which notably include Personal Factors. For the reintegration into employment in general or a specific work place, an extensive evaluation of Personal Factors may be crucial.

\section{An Appropriate Consideration of Personal Factors is Necessary for A Comprehensive Needs Assessment}

A professional assessment of the type and dimension of restrictions and resultant participation goals comprehensively describes the functioning of an individual only if Environmental and Personal Factors are considered and integrated into the conclusions (as indicated by the ICF). Thus, transparent reporting, e.g., of work capacity evaluations, would be the basis for a more comprehensible decision regarding eligibility benefits. On this basis, appropriate needs are identified, adequate interventions are initiated and suitable service providers are chosen to effectively match the participation goals.

The use of a standardised description for the documentation of Personal Factors would facilitate and improve the interdisciplinary understanding and communication across all collaborating institutions and sectors.

Personal attitudes, competencies, desires, strengths and weaknesses become more evident and may be included into recommendations and decisions, with a focus on the impacts on an individual's health problem and life background. The quality of allocation, procedures and goal 
achievement will improve. It is advantageous if the assessment and decisions of professionals, such as medical and social experts, are acquainted with a commonly accepted classification of Personal Factors.

\section{A Classification of Personal Factors is Not an Assessment Instrument}

WHO established ICF as a classification, not as an assessment instrument. Thus ICD 10 cannot replace a clinical examination, sonography or laparoscopy, and ICF cannot substitute for an assessment. However, assessment findings may be arranged in order and codified using a classification.

Assessment instruments typically comprise standardised test procedures for circumscribed items, the results of which are primarily measured and summed using scaled numerical values. Regarding Personal Factors, the perspective of an evaluation is quite different. The quantitative deviation from normality is not of interest; however, the answer to the generaI question of their positive or negative qualitative influence on the health condition and its impact on the other components of functioning as facilitators or barriers to a specific health condition may be relevant. For example, the characteristic of an individual irrevocably guided by specific principles may be a facilitator for an occupation in the final checking area, whereas the same characteristic may be a barrier for social relations.

Given this assumption, Personal Factors must be well circumscribed and definable. Specific items may be determined using measuring methods only when it is of further interest or necessary to solve a specific problem. However, one fact is obvious: There is no measurement method to reliably determine whether an item acts as a facilitator or a barrier in an individual case. This determination is only possible by applying a holistic view with a specific question respective to a specific individual.

\section{Individuals with Disabilities Should be Involved in all Aspects of the use of Personal Factors}

Representatives of organisations for individuals with disabilities should actively contribute to the process of discussions regarding Personal Factors. Thus, the accuracy and acceptability of the present draft for the conceptual design of the missing ICF component may be promoted and its structure considered feasible for real life application.

The inclusion of Personal Factors in interviews / anamnesis of involved individuals / patients and their affiliated individuals enables therapists and physicians to obtain a closer view of the needs of the individuals concerned and their desires, strengths and weaknesses, as well as to include the findings in their expert opinions. An orientation to the needs of individuals would promote better, goal-oriented rehabilitative interventions, and social benefits would receive a qualitative boost. The consideration of Personal Factors will furthermore stimulate self-determination and equal-rights based participation in social life. Therefore individuals must be included into the assessment process. 
However, the requests of the authors in the generation of the draft of Personal Factors towards associations or unions of individuals with disabilities and their umbrella organisations have addressed problems to efficiently activate the participation of involved individuals. Irrespective of this problem, individuals with disabilities attentively observed developments and discussions regarding the ICF and especially regarding Personal Factors. They appraise the potentials of a transparent and cross-sectoral needs assessment quite variably. Acceptance ranged from active participation to preparation of an instruction tutorial for users of the ICF by maintaining an expectant distance up to an open rejection. The reasons for a rejection are the inter alia negative experiences of individuals with inadequate assessments in their own history.

Involved individuals do not always agree with the expert's opinion and often argue that their view on the type and extent of participation restrictions has not been adequately respected. The expert is more often viewed as a lobbyist mandated by the benefit agency than a law-abiding expert committed to objectivity. The acceptance of involved individuals to systematically consider relevant Personal Factors will only be achieved by indicating and convincing them that this approach is an instrument to provide transparency for individually driven decision processes by the benefit agencies. Finally, it will make the justiciable enforcement of entitled claims for benefits easier [6].

It is presupposed that Personal Factors are explicitly and transparently reported and not in a hidden manner. This allows a claimant to understand a medical report and formally object at the court in case he deems a statement or decision by the expert or deciding body to be unwarranted.

\section{The use of Personal Factors Accounts for Ethical Aspects, Specifically, the Guidelines to use ICF}

The ethical tenor to classify Personal Factors is demonstrated by the broad selection of criteria: The categories and items should be comprehensive, universally applicable, unprejudiced, nondiscriminatory and clear without ambiguity. They should be practically usable, limited to relevant categories and aligned to a final (not causally) determined sociomedical view. Furthermore, the involved individual with his / her right for autonomy and participation must stand in the centre.

The potential consequences of listing Personal Factors must be weighed with their usefulness in mind, as well as the conceivable risks and judicial aspects. Ethical considerations must distinguish between descriptive and normative levels. The proposed list does not express a normative character in the sense of a moral attribution to specific factors. Instead, Personal Factors are described and arranged within the scope of a respective question that refers to their effects on functioning and disability as a facilitator or barrier. The draft supports the neutral description of these influential factors.

The WHO has established eleven ethical guidelines for the use of the ICF that reflect respect of the autonomy of individuals whose disabilities are described using the ICF. The first guideline claims that the "ICF should always be used to respect the inherent value and autonomy of individual persons". The fourth guideline addresses data protection: "The information coded 
using ICF should be viewed as personal information and subject to recognised rules of confidentiality appropriate for the manner in which the data will be used."

Guidelines 8 to 11 consider the social dimension:

"8. ICF information should be used, to the greatest extent feasible, with the collaboration of individuals to enhance their choices and their control over their lives.

9. ICF information should be used towards the development of social policy and political change that seeks to enhance and support the participation of individuals.

10. ICF, and all information derived from its use, should not be employed to deny established rights or otherwise restrict legitimate entitlements to benefits for individuals or groups.

11. Individuals classed together under ICF may still differ in many ways. Laws and regulations that refer to ICF classifications should not assume more homogeneity than intended and should ensure that those whose levels of functioning are being classified are considered as individuals".

To provide an explanation, table 2 presents contrasting ethical statements.

Table 2: Ethical Statements for the Respect of Individuals

\begin{tabular}{|l|l|}
\hline Not of primary Interest: & The Questions to be solved: \\
\hline $\begin{array}{l}\text { How broad is the capacity of } \\
\text { action of an individual? }\end{array}$ & $\begin{array}{l}\text { Is the capacity of an action of an individual sufficient for a } \\
\text { designated intervention or procedure, e.g., the use of an } \\
\text { auxiliary means, or does the person require other help or } \\
\text { support? }\end{array}$ \\
\hline $\begin{array}{l}\text { Which religion or political credo } \\
\text { does the individual belong to? }\end{array}$ & $\begin{array}{l}\text { Are there influences on the functioning or disability that } \\
\text { result from the philosophy of life (religion, politics), e.g., a } \\
\text { custom for a specific food? }\end{array}$ \\
\hline $\begin{array}{l}\text { Which language(s) does the } \\
\text { individual speak? }\end{array}$ & $\begin{array}{l}\text { Is there a language knowledge for speech competence and } \\
\text { linguistic communication that affects the deliberate } \\
\text { processing of information, including understanding the } \\
\text { relevance of what is perceived and placing it into an } \\
\text { intelligent context? }\end{array}$ \\
\hline $\begin{array}{l}\text { Which nationality does the } \\
\text { individual belong to? }\end{array}$ & $\begin{array}{l}\text { Are there behavioural patterns, cultural habits or attitudes } \\
\text { as a result of repetition by growing up in another country / } \\
\text { nation that have become routine and serve as barriers or } \\
\text { facilitators when considering help for participation? }\end{array}$ \\
\hline
\end{tabular}

A medical doctor should be attentive to the religion of his patient. It would be unprofessional to disregard this important personal factor. During fasting, medical treatment of patients is often a challenge for medical doctors: Not only medical knowledge but also information regarding the rules of faith and a respectful attitude are necessary. It is well known that many patients stop taking medication throughout the day or take the complete dose once before sunrise or after sunset even though sick persons are often exempt from the obligation to fast. The knowledge of this information may indicate the need for changes in the medication to a prolonged release formulation, adjustment of the pharmaceutical form (e.g., use transdermal plasters, sprays, topical creams or injections, which may be compatible with the rules) or consideration of the bioavailability and pharmacokinetics in relation to eating habits. 


\section{Classification may be Associated with Risks}

Personal Factors represent a logical and fundamental approach for the application of the BioPsycho-Social Disease Model. As components of contextual factors, in general, they exert the same influences as Environmental Factors.

To date, the WHO has not classified Personal Factors "because of the large social and cultural variance associated with them". These overall difficulties do not prevent consideration of Personal Factors on an individual basis or the arbitrary use or handling of them.

The listing of Personal Factors and drafting them into a classification is of cognitive interest and neutral value. However, to intend or actually use these factors involves risks and raises fears. Therefore, in each case, responsible handling of a systematic compilation of Personal Factors is crucial and unconditionally necessary.

As previously stated, the appropriate analysis and evaluation of relevant Personal Factors eases a needs-oriented assessment of their facilitating or inhibiting influence on the functioning or disability of an individual. The opportunity to select an individual and accurately fit social and medical participation benefits with respect to self-determination is thereby promoted.

Nevertheless, in some cases, Personal Factors may not be used appropriately and professionally, they are assessed in their range unreasonably or their facilitating or inhibiting influence on the functioning of an individual in terms of the ICF is missed. Promising prospects may remain unused, and the risk may also result in the selection of inappropriate social or medical benefits or unjust refusal as a result of overlooking critical Personal Factors.

The use of Personal Factors with levity in clinical everyday life may represent a risk because of an unreflected stereotyped thinking regarding general statements that concern an individual instead of focusing on a specific circumstance. Moreover, demarcation problems may arise because of differences that are not always distinguishable on the first view between the items of the classification of Personal Factors or compared with the other remaining ICF components. However, this issue appears to be less relevant for use in clinical and sociomedical daily life.

Furthermore, users may have difficulties with discriminations in contents and terminology, as well as for reasons related to data protection. The collection, validation, documentation and forwarding of data must comply with the respective legal allowances.

Personal Factors may only be used to answer a specific question aiming at a specific decision at a specific time. Therefore, their attribution is of limited validity and is not transferable to other situations. The remaining subjectivity of the cognitive assessment act is not critical as long as all involved individuals are informed transparently and with a mutual understanding. It is critical that Personal Factors are appraised only in context with the requested social and medical benefits by an expert who uses a qualitative approach that aims to identify factors that are facilitators or barriers in their effects on functioning and disability. The draft for the classification of Personal Factors does not contain or allow a quantitative approach. 
In a comparison of the relevant pros and cons, it is undoubtable that the unsystematic, intransparent, inconsistent and incommensurable non-standardised use of Personal Factors is of high risk in the assessment of evaluations or decisions that are perceived of as unfair by the claimant.

\section{Motivation is a Complex Construct and Requires Sophisticated Consideration}

The terms "existing or non-existing motivation" for a proposed intervention are repeatedly occurring in medical statements as a personal attribute. This is a very meaningful example about the necessity to clearly describe Personal Factors and demarcate them from others. Motivation as a symptom of a psychological disorder (e.g., depression) must be distinguished from a lack of motivation as a personal attitude (i.e., a Personal Factor), which is discussed here. Existing or missing "Motivation" is an important question if not the central point for the allocation of many medical interventions and social benefits to avoid, alleviate or remove the impacts on functioning and disability. However, motivation is a construct, which consists of emotional factors, available energy, impulse, empowerment, and proaction. Terms such as motives, readiness to act, pursuit of effectiveness, self-management, ambition, impulse, desires, impetus, lust, objectives, will and self-determination are often linked with the term motivation. Miscellaneous modes of motivation distinguish between extrinsic and intrinsic motivations as sources of motivation.

The question of whether an individual is willing, ready and mentally able to initiate rehabilitation should be independently and comprehensively assessed by the evaluating expert. Where applicable, a summary of the findings that indicate, for example, an individual "is not motivated for rehabilitation" must not be apprehended as a persisting characteristic of the individual.

Thus we decided not to describe the complex attribute "motivation"; instead, it serves as a basis for a broader depth of underlying items. Therefore, the corresponding items are presented in chapter 3 (mental factors) and chapter 4 (attitudes, basic competencies and habits of behaviour). By applying this model, attitudes and moral concepts (which undoubtedly are components of an integrated concept of motivation) may be noticed as facilitators or barriers, and the interactions between specific factors may be demonstrated.

\section{The use of Personal Factors should be Tested in Practice, as well as Scientific Analyses}

The draft for Personal Factors of the DGSMP workgroup is an initial step. It should now be tested in practice. The clarification of several questions requires a scientific analysis.

\section{Common understanding and scientific foundation of differing terms}

Personal Factors, which represent a central and significant value in health sciences, should be elucidated in a manual. This approach ensures that all individuals who handle the ICF dispose of a uniform understanding of Personal Factors, which is imperative for a standardised procedure to enable scientific analyses. These may comply with comparative analyses, e.g., to determine the retrieval of multicentre concurrent terms used to describe specific dysfunctions with specific Personal Factors of central distinctiveness and to verify hypotheses regarding whether a Personal Factor is a facilitator or a barrier in the context of a specific health condition. 
A classification must be adequate not only to reliably serve all differentiations (e.g., no overlapping of terms and constructs) but also to be consistent with a claimed completeness. The goal of the presented list is and was to cover all relevant Personal Factors. Therefore, an analysis of respective publications is also of value for future scientific work even if they utilised approaches with different methods. A comparison of eight categorisations that substantially differ in their backgrounds, development and structures (Müller and Geyh 2014) identified a common core of content issues to build the basis for further development (socio-demographic factors, behaviour / lifestyle, cognitive / psychological factors and coping). This analysis also described additional content areas that were explicitly not included in the ICF definition of Personal Factors, such as social relationships, aspects of emotions, biological / physiological factors and motives / motivation, which should be further examined and discussed. Importantly, the construct-related and criterion-related evidence for the validity of our draft, which was included in the previously described comparison, should be qualitatively (e.g., via a Delphi procedure) and quantitatively assessed. The latter is only possible after the presentation of appropriate data. This approach will lead to the next challenge.

\section{Applied Research}

For practical use (e.g., in rehabilitation), a recommendation is needed regarding how to evaluate and document Personal Factors. A qualitative analysis of the manageability for assessments in practice is desirable, e.g., by conducting half-standardised interviews. By generating questiondriven shortlists, the user may be motivated to handle Personal Factors in a structured consideration.

The handling of the inclusion coverage and documentation of Personal Factors would be of interest with respect to the level of information for the individuals involved. Considering the new initiatives of health care with an increased involvement of concerned individuals, Personal Factors are also of interest. Specifically, this will be necessary for the increasing number of elderly, often multimorbid, individuals, as well as individuals with chronic health problems and in respect to age and gender. Furthermore, if considered possible, applied research should be conducted by engaging self-help organisations.

\section{Theory-driven Research}

As soon as sufficient data are available, multivariate analyses may be applied to test the BioPsycho-Social Model and its interactions between specific components of the ICF. Personal Factors and their influence on functioning, as well as the underlying type and extent of interaction may be of interest. Moreover, the relationships and interactions between specific Personal Factors regarding their cumulative effects as barriers or facilitators are also critical. Another activity may pursue the question of whether interactions exist between factors of risk and factors of resilience (barriers and facilitators in the ICF, respectively) by mitigating the impact of risk factors on the health condition and / or strengthening resilience factors. 


\section{Discussion and Limitations}

It is the task of physicians who practice Social/ Insurance Medicine to assess the prerequisites for sociomedical benefits, such as sickness allowance, medico-vocational or pure medical rehabilitation, auxiliary means or invalidity pensions granted by insurances or social security agencies. It may be important for decisions, such as those concerning adequate medicovocational rehabilitation, to meet individual vocational aptitudes and interests, vocational or school qualifications, language skills, specific competences or convictions. Sociomedical benefits must be tailored to suit personal needs to be successful, while simultaneously considering the determining framework of the particular health insurance / social security system. The underlying assessment must respect and consider the individual life situation of the individuals involved. There is a clear position by professionals who act in different branches of Social / Insurance Medicine that the finding and allocation of promising benefits appropriate for an individual's needs must consider Personal Factors as barriers or facilitators. As a consequence, we officially implemented our classification of Personal Factors as a helpful tool for medical experts who practice social and insurance medicine to consider Personal Factors in their expert reports when assessing the needs of disabled individuals or recommending potentially successful interventions for the German Social Health Insurances.

Nevertheless, reflecting on recent literature, especially the remarks of Simeonsson et al. 2014 [5], Müller et al. 2014 [6] and Leonardi et al. 2015 [7], questions remain to be solved and discussed, which this paper hopes to promote: We presented a definition of terms to be used as a starting point for a subsequent debate. Moreover, it is clear that substantial work remains to be done. We agree that the definition of items listed as Personal Factors must be as clear, precise and demarcateble as possible. At the same time, we must be aware that a unique accordance over different professional guilds regarding specific factors, e.g., mental factors or attitudes, basic skills and behaviour patterns, will not be reached within years, if at all. However, we must also consider that the current situation in Social and Insurance Medicine as a branch of Social Medicine is quite different from the scientific aims and processes. We do not have the time to wait for the results of another 15 years of research (ICF was published by the WHO in 2001!). If we agree that an item such as "character", which is a term that we purposely do not use in our model as some authors do, is necessary to be considered for sociomedical decisions, we would consensually and scientifically be most interested in defining this item as exact as possible, without overlapping with other ICF-Items, and enabling its measurement using a scale. However, in Social / Insurance Medicine, the situation is different: we are not practically interested in examining the individual character under a microscope and determining the degree of deviation from an average population measure. We aim to assess the impact of Personal Factors on a health problem, to solve a specific question at a specific time to prepare a decision for specific social benefits; however, it is not intended to describe the so called character in detail or in general or the value of it. At the end of our task, we must come to a conclusion, i.e., whether the individual sitting in front of us with his or her inherent Personal Factors and their impact on the individuals functioning is suitable to work, e.g., as a pilot. A decision must be made by holistically examining the individual according to the bio-psycho-social disease model, performing a task-oriented evaluation and documenting the relevant results for the question to be solved and being certain about the informed consent of the individual. 
This leads to the necessity to discuss methods and procedures (anamnesis, sense of self, awareness of others, and underpinning psychological tests) to assess the influence of Personal Factors on functioning and how the results may or should not be documented. In practical Social / Insurance Medicine, we are interested in qualitative Personal Factors that inhibit or promote promising sociomedical benefits. A statement regarding the quantitative deviation of items, such as extraversion of openness from the average population, is not helpful to solve the previously described questions. Thus, it is doubtful, in this example, whether a character's mapping would be ethically approved, although it may have been correctly measured scientifically. We would strike the borderline of science. It is doubtful whether a character should be quantified at all. Therefore, guidelines that advise when and how to correctly document Personal Factors that influence decisions regarding particular sociomedical benefits would be helpful. These guidelines would help to prevent the misuse of Personal Factors in the initiation of unwarranted decisions and statements as collateral damage.

The influence of Personal Factors on the health and life stories of individuals and their compatibility using ICF terms has been confirmed, to a substantial extent, over the years by Dür et al. 2015 (rheumatoid arthritis [8]), Hoefsmit et al. 2014 (return to work [9]), Rapp and Queri 2016 (oncology (10)), Niek Stoop 2014 (daily practice of the General Practitioner [11]), Schwegler et al., 2013 and 2014 (medical work capacity evaluations, chronic widespread pain and low back pain, psychiatry in social medicine [12-14] Offenbächer et al. (mindfulness [15]), Hamed et al. 2013 (multiple sclerosis [16]), Howe 2008 (speech-language pathology [17]) and Heerkens 2004 (occupational medicine [18]). However, it appears that compatibility with ICF taxonomy is accompanied by the need for items not currently listed in the ICF. This leads to the fact that context-specific additions are necessary for comprehensive and transparent reporting in sociomedical reports, which indicates the need for a discussion to continue the search for a list of Personal Factors within the ICF frame or beyond. This list would help to memorise the nature and diversity of Personal Factors likely relevant for the question to be solved but not to be worked off from A to Z. If using the tool of the ICF, this list may have items to describe temporary health related functional deficits, while also applying the same terms as long-lasting Personal Factors, as performed by some authors, which may also be considered to be the solution for a taxonomically incorrect understanding of the ICF. We suggest that Personal Factors should stand alone and should be distinguished from disease-induced impacts on personality. Without doubt, this is a difficult job to accomplish, and, in many cases, it may be comprehensively impossible without an extensive biographical anamnesis, as practicing physicians are aware.

\section{Conclusion}

The potential to promote a uniform investigation of Personal Factors is not sufficiently supported by the ICF to date. However, the knowledge of relevant Personal Factors in individual cases is of substantial importance for the rehabilitation process, as well as the allocation of needs oriented and accurately fitting benefits for chronically diseased and elderly multimorbid individuals. Personal Factors may exert a powerful influence as facilitators or barriers on the goal definition, goal achievement and type, complexity and length of necessary social or rehabilitation benefits. Transparency, explicitness, fairness (standardisation) and the possibility for well-founded claims comprise additional arguments for reporting Personal Factors, as well as key aspects that should be considered when applying a standard classification of Personal Factors in Social Insurance 
and Security. Thus, Personal Factors may be crucial to reach the goal of the highest degree of participation in social life considering individual resources.

Therefore, a uniformly accepted classification is necessary. The presented draft of a list of Personal Factors is generated in the absence of a systematic structure of Personal Factors by the WHO. The suggested items and definitions may serve as tools to describe conditions that, in general, are understandable. It promotes a uniform understanding independent of a specific professional jargon. It is advised for use by all individuals involved in the processes of social integration and eligible to reduce the risk of various approaches to Personal Factors.

To comprehensively settle the needs benefits, the generation of a classification system is not only legally permitted and obvious but also necessary. As a matter of course, the legal and ethical norms, including the data protection regulations, is to be accounted for in the same way as is the case with all other procedures for needs assessment, specifically, the involvement of the individuals affected.

Moreover, a standardised foundation to assess Personal Factors will enable a comprehensive application of the Bio-Psycho-Social Model and the interactions between the components of the ICF. All components of the ICF may thus be systematically integrated into clinical / sociomedical expertise reports.

The explicit reference to rehabilitation or participation planning with the inclusion of relevant Personal Factors induces a dialogue function between the rehabilitation team and the involved individual as an impulse for the treatment options. Different appraisals between therapeutic personnel, medical doctors and rehabilitated individuals may be discussed and recorded. This approach may lead to a differentiated point of view and may induce a learning effect in the longterm. Provided relevant Personal Factors are assigned to the participation benefits, the communication with the funding statutory agencies may be facilitated regarding Personal Factors and their impact on rehabilitation relevant effects.

The use of Personal Factors in Practical Social Medicine is not innovative. However, their present use is often unstructured, unsystematic, inconsistent and, in some cases, incomprehensible as a result of non-standardised wording. Therefore, having a list of generally accepted Personal Factors in mind and using their items is feasible, practicable and not time consuming.

The proposed draft of a classification of Personal Factors aims to provide a foundation for scientific studies regarding their importance for the rehabilitation and reintegration of disabled individuals with their individual health conditions into working life.

We have established a list of Personal Factors within the ICF frame; however, we also consider it worthwhile to subsequently assess the pros and cons of whether it is inevitable to classify Personal Factors using the ICF framework or to discuss an alternative construction of Personal Factors beyond the restrictions of the ICF as proposed to question by Leonardi et al. [7]; an alternative approach may thereby avoid the overlapping contents of components (as is the case with activities and participation as well) and the necessity to link Personal Factors to ICF- 
taxonomy. Moreover, the concentration on the bio-psycho-social model as an anchor may be sufficient. Thus, we appreciate the need to explicitly discuss the question raised by Simeonsson et al. as to whether an additional "Box" is even needed in the ICF.

\section{Financial Support}

None.

\section{Declaration of Interest}

The authors report no declarations of interest.

\section{References}

[1] World Health Organization. The International Classification of Functioning, Dis-ability and Health (ICF). WHO 2001. Geneva. Available from: http://www.who.int/classifications/icf/en/

[2] Antonovsky A. Health, Stress and Coping, Jossey-Bass. San Francisco: 1979.

[3] Grotkamp S, Cibis W, Nüchtern E et al. Personal Factors in the International Classification of Functioning, Disability and Health: Prospective Evidence. Aus-tralian Journal of Rehabilitation Counselling 2012; 18 (1): 1-24.

[4] Grotkamp S, Cibis W, Nüchtern E et al. 2012. Personbezogene Faktoren der ICF - Beispiele zum Entwurf der AG „ICF“ des Fachbereichs II der Deutschen Gesellschaft für Sozialmedizin und Prävention (DGSMP). Gesundheitswesen 2012; 74: 449-458.

[5] Simeonsson R J, Lollar D, Björck-Akesson E, Granlund M, Brown S C, Zhuoying Q, Gray D and Pan Y. (2014) ICF and ICF-CY lessons learned: Pandory`s box of personal factors, 36 (25),2187-94

[6] Müller R and Geyh S. (2015 Lessons learned from different approaches towards classifying personal factor. Disab and Rehabil, 2015; 37 (5):430-8

[7] Leonardi M, Sykes C R, Madden RC, Ten Napel H, Hollenweger J, Snyman St, Madden R H, De Camargo O K, Raggi A, Van Gool C H, Martinuzzi A and on behalf of the Functioning and Disability Reference Group of the WHO-FIC. Do we really need to open a classification box on personal factors in ICF? Disab and Rehabil 2016 ; 38 (13): 1327-8

[8] Dür M, Coenen M, Stoffer M A, Fialka-Moser V, Kautzky-Willer A, Kjeken I, Dragoi R G, Mattson M, Boström C, Smolen J and Stamm T A 2015. Do patient-reported outcome measures cover personal factors important to people with rheumatoid arthritis? A mixed methods design using the International Classification of Functioning, Disability and Health as frame of reference. Health and Quality of Life Outcomes 2015: 1-13

[9] Hoefsmit N, Houkes I and Nijhuis F. Environmental and personal factors that support early return-to-work: A qualitative study using the ICF as a framework. Work 2014; 48: 203-215

[10] Rapp RS and Queri S. Validierung der Personbezogenen Faktoren im Bereich der onkologischen Brustkrebsrehabilitation - eine Delphi-Befragung auf ationaler Ebene. Gesundheitswesen 2016; eFirst

[11] Stoop N. Registration of personal factors of patients in the general practice: which are important to register according to general practitioners? Radboud University Medical Center, RUMC, Department of Primary and Community Care 2014: 1-36

[12] Schwegler U, Peter C, Trezzini B, Anner J, Geyh S. Toward transparent documen-tation in medical work capacity evaluations: identifying personal factors in medical reports on Swiss disability claimants with chronic widespread pain. International Journal of Rehabilitation Research, 2013; 36 (4): 298-307. 
[13] Schwegler U, Anner J, Glässel A, Brach M, De Boer W, Cieza A and Trezzini B. Towards comprehensive and transparent reporting: centext-specific additions to the ICF taxonomy for medical evaluations of work capacity involving claimants with chronic widespread pain and low back pain. BMC Health Services Research 2014; 14, 361: 1-13

[14] Schwegler U, Peter C, Anner J, and Trezzini B. Toward standardised documenta-tion in psychiatric evaluations: Identifying functioning aspects and contextual fac-tors in psychiatric reports of Swiss disability claimants with chronic widespread pain. Swiss Med Wkly. 2014; 144: w14008.

[15] Offenbächer M, Sauer S, Hieblinger R, Hufford D J, Walach and Kohls. Spirituality and the International Classification of Functioning, Disability and Health: content comparison of questionnaires measuring mindfulness based on the International Classification of Functioning. J Disabil Rehabil 2011; 33 (25-26):2434-45

[16] Hamed R, Abu Tariah H and Hawamdeh Z M. Personal Factors Affecting the Daily Functioning and Well-Being of Patients with Multiple Sclerosis Using the International Classifcation of Functioning Model. International Journal of Mental Health 2013; 41 (4): 47-61

[17] Howe $\mathrm{T}$ J.The ICF Contextual Factors related to speech-language pathology. In-ternational Journal of Speech-Language Pathology 2008; Vol. 10 (1-2): 27-37

[18] Heerkens Y, Engels J, Kuipers Ch, Van der Gulden J and Oostendorp R. The use of the ICF to describe work related factors influencing the health of employees. Disability and Rehabilitation 2004; 26 (17): 1060-1066

*Corresponding author.

E-mail address: wolfgang.seger@ mdkn.de 